\title{
Démarche intégrative dans les écoles de thérapie de la Charte suisse de Psychothérapie
}

\author{
Aureliano Crameri, Margit Koemeda, Volker Tschuschke, Peter Schulthess \& Agnes von Wyl \\ Psychotherapie-Wissenschaft 8 (2) 83-84 2018 \\ www.psychotherapie-wissenschaft.info \\ CC BY-NC-ND \\ https://doi.org/10.30820/8243.15
}

Mots-clés : Intégration-psychothérapie, techniques psychothérapeutiques, formation thérapeutique, processus-psychothérapie Q-Set, PAP-S-Rating-Manual

Dans la deuxième moitié du siècle dernier, le nombre de procédés thérapeutiques a augmenté d'une manière exponentielle. En même temps que cet accroissement, une pratique éclectique s'est également propagée avec une réduction conséquente de la fidélité envers les procédés thérapeutiques classiques. Différents sondages auprès de psychothérapeutes ont démontré qu'ils n'étaient qu un petit pourcentage à se tenir à une seule méthode, tandis que la plupart d'entre eux s'identifient à plusieurs orientations thérapeutiques ou se caractérisent comme éclectiques. Ce phénomène est désigné comme intégration ou multi-modalité et s'applique aujourd'hui comme un leitmotiv dans la psychothérapie. Un argument des thérapeutes en faveur d'une approche intégrative est la possibilité de pouvoir sélectionner en fonction de la situation la meilleure technique possible pour chaque patient.

L'intégration n'est pas seulement définie sur un niveau thérapeutique technique, mais également à un niveau d'abstraction plus élevé, par ex. avec l'acceptation de principes communs de fonctionnement (" common factors »), qui sont sous-jacents aux différentes méthodes thérapeutiques. Ce postulat est souvent étayé par des résultats de méta-analyses qui renvoient à une équivalence de l'efficacité de différentes méthodes thérapeutiques. L'étude de cas Psychothérapie ambulatoire suisse (PAP-S) a également apporté des preuves d'une équivalence d'effets. En outre, l'analyse d'enregistrements audio de séances sélectionnées aléatoirement de cette étude a révélé que seule une partie relativement faible des interventions utilisées provenait de la formation thérapeutique spécifique de chaque thérapeute, tandis qu'au moins la moitié devait être classifiée comme des interventions générales.

Ces résultats ont soutenu la thèse selon laquelle la majorité des thérapeutes exerçaient une pratique intégrative. Hormis ces exceptions, des points communs dans la posture thérapeutique ou dans l'application de techniques thérapeutiques par des techniciens thérapeutiques entre des thérapeutes de différentes écoles ont été analysés dans le présent article sur la base d'enregistrements audio provenant de l'étude PAP-S. Deux échantillons de 108 et 162 séances issues de différentes thérapies ont été utilisés comme base de l'analyse et évalués à l'aide de l'un des deux systèmes de notation standardisés.
Le premier système de notation était le Q-Set processus-psychothérapie qui permet de décrire et de classifier l'interaction entre le thérapeute et son patient. Avec le Q-Set, un échantillon de 54 séances de la Psychothérapie intégrative du corps (IBP) a été comparé avec un échantillon de même taille de séances de la Psychothérapie orientée processus (IPA). Les deux concepts thérapeutiques bien que différemment définis contiennent des éléments issus de la Psychologie du profond, de la Psychologie humaniste et de la Psychothérapie du corps et doivent être tous les deux classifiés comme intégratifs. L'approche intégrative des représentants des deux écoles a été confirmée par l'analyse des caractéristiques de processus à l'aide du Q-Set : Sur le plan de l'organisation des séances de thérapie, il est apparu une concordance de 80 pour cent entre les deux groupes de thérapeutes. La partie substantielle de postures et de traitements cognitifs-comportementaux non explicitement définis dans les concepts thérapeutiques correspondants était toujours intéressante.

Le deuxième système de notation sur l'analyse du traitement thérapeutique était le PAP-S-Rating-Manual, qui a été conçu dans le contexte de l'étude pour identifier différentes interventions générales et psychothérapeutiques spécifiques aux écoles. Dans cette deuxième évaluation, les interventions psychothérapeutiques ont été dénombrées et catégorisées dans un échantillon de 162 séances au total de thérapeutes issus de six différentes écoles. Il a été possible de créer les cinq catégories suivantes : des interventions liées aux émotions, des interventions liées au comportement, des interventions orientées sur l'inconscient, des interventions liées au corps et des interventions orientées sur l'art et l'expression. Chaque catégorie d'intervention a été observée au moins sur un thérapeute de chaque institut. La signification des interventions liées au comportement a également été confirmée ici. Sur le plan de la fréquence, ce type d'interventions arrive en deuxième position après les interventions liées aux émotions. Ces résultats suggèrent que les concepts psychodynamiques et humanistes à un niveau d'intervention convergent en partie vers une approche orientée sur le comportement, qui est traditionnellement attribuée aux approches cognitives-comportementales.

Il était également intéressant de constater que des techniques aussi se concentrant sur des niveaux " non verbaux ", 
comme des interventions orientées sur l'art et l'expression ont été observées dans chaque groupe de thérapeutes.

Globalement, les résultats indiquent qu'une approche intégrative est répandue à la fois à un niveau institutionnel et personnel. Dans certaines écoles, l'intégration-psychothérapie est déjà ancrée dans leur concept thérapeutique. En outre, les thérapeutes avec une expérience professionnelle croissante à un niveau personnel pratiquent une intégration assimilative, en reprenant également des techniques et des actions issues d'autres approches thérapeutiques. Une raison qui justifie le développement d'une approche intégrative est l'expérience faite par des thérapeutes selon laquelle une seule méthode thérapeutique a ses limites et ne montre pas sur tous les patients l'effet attendu.

\section{Les auteures et auteurs}

Aureliano Crameni, Dr. phil. est responsable qualité, collaborateur de recherche dans le groupe professionnel Psychologie clinique et Psychologie de la santé à l'École supérieure de Zürcher de Sciences Appliquées, Département Psychologie Appliquée, ainsi que professeur en méthodes de recherche.

Margit Koemeda-Lutz, Dr. Dipl.-Psych., est psychothérapeute (ASP), superviseuse en psychothérapie et formatrice (SGBAT, IIBA), ainsi que membre de la commission scientifique de la Charte suisse de Psychothérapie. Par ailleurs, elle est active dans trois revues en tant que membre de la rédaction, auteure de nombreux ouvrages et articles spécialisés sur des thèmes de la recherche en psychothérapie et la psychothérapie du corps.

Volker Tschuschke, Univ. Prof. Dr. Dipl. Psych, est un ancien détenteur de chaire émérite de la spécialité Psychologie médi- cale à la Clinique universitaire de Cologne. Il est psychanalyste et a été pendant des années professeur auprès de différents instituts de formation, est l'auteur de nombreux ouvrages et contributions dans des revues spécialisées internationales et nationales et travaille par ailleurs comme psychothérapeute et superviseur.

Peter Schulthess, MSc, psychothérapeute au niveau fédéral (ASP). Depuis de nombreuses années formateur en Gestaltthérapie en Suisse et au niveau international. Ex-président de la Charte suisse pour la psychothérapie, actuellement membre du comité de l'ASP. Chair du "Science and Research Committee " de l'EAP. Rédacteur en chef de la revue PsychotherapieWissenschaft et auteur ou co-auteur de diverses publications sur la psychothérapie et Gestalt-thérapie.

Agnes von Wyl, Prof. Dr., est directrice du groupe spécialisé Psychologie clinique et Psychologie de la Santé à l'école supérieure de Zürcher de Sciences Appliquées, Département Psychologie Appliquée. Elle travaille par ailleurs en tant que professeure et psychothérapeute psychanalytique. Ses domaines de recherche comprennent la recherche en psychothérapie, Infant Mental Health et la promotion psychique de la santé.

\section{Contact}

Aureliano Crameri

ZHAW Zürcher Hochschule für Angewandte Wissenschaften Departement Angewandte Psychologie

Pfingstweidstrasse 96

Postfach 707

8037 Zurich

E-Mail : aureliano.crameri@zhaw.ch 